\title{
Correlates of bacterial ulcers and acute HSV-2 infection among men with genital ulcer disease in South Africa: age, recent sexual behaviours, and HIVi,ii
}

\author{
Jami S. Leichliter**, David A. Lewis ${ }^{c, d, e, f}$ and Gabriela Paz-Bailey ${ }^{b}$ \\ ${ }^{a}$ Division of STD Prevention, Centers for Disease Control and Prevention, Atlanta, GA, USA \\ ${ }^{b}$ Division of HIV/AIDS Prevention, Centers for Disease Control and Prevention, Atlanta, GA, USA \\ 'Centre for HIV and STIs, National Institute for Communicable Diseases, National Health Laboratory Service, Johannesburg, South Africa \\ ${ }^{d}$ Department of Internal Medicine, Faculty of Health Sciences, University of the Witswatersrand, Johannesburg, South Africa \\ eWestern Sydney Sexual Health Centre, Parramatta, Australia \\ ${ }^{f}$ Centre for Infectious Diseases and Microbiology \& Marie Bashir Institute for Infectious Diseases and Biosecurity, Westmead Clinical School, \\ University of Sydney, Sydney, Australia \\ *Corresponding author, email: jleichliter@cdc.gov
}

Data from baseline surveys and STI/HIV laboratory tests ( $n=615$ men) were used to examine correlates of bacterial ulcers (Treponema pallidum, Haemophilus ducreyi, or Chlamydia trachomatis L1-L3 detected in ulcers) and acute HSV-2 ulcers (HSV-2 positive ulcer specimen, HSV-2 sero-negative, and negative for bacterial pathogens) versus recurrent HSV-2 ulcers (seropositive), separately. Men with bacterial ulcers had larger ulcers compared to men with recurrent HSV-2 ulcers, but were less likely to be HIV-positive; whereas, men with acute HSV-2 ulcers were younger with fewer partners. Acute HIV was higher among men with bacterial and acute HSV-2 ulcers; however, this difference was not statistically significant.

Keywords: acute HIV, acute HSV-2, bacterial ulcers, genital ulcer disease, sexual behavior

\section{Introduction}

Genital ulcer disease (GUD), including herpes simplex virus type 2 (HSV-2) infection, is common in sub-Saharan Africa; and, is associated with HIV acquisition and onward transmission. ${ }^{1-6}$ Although some studies have examined demographics and sexual behaviours of men with GUD, ${ }^{1}$ less is known about the differences between men with bacterial and other acute ulcers and men with other forms of GUD. Given the relationship between HSV-2 and HIV acquisition ${ }^{7}$ and the difficulties in the ability to clinically differentiate between specific etiological causes of GUD ${ }^{8}$, it is important to examine whether or not men presenting to a clinic with GUD have any demographic or sexual risk differences based on ulcer etiology. Identifying clinical, demographic and behavioral correlates of GUD by causal infection may aid in the development of targeted counselling messages regarding STI/HIV acquisition and transmission risk as well as STI/HIV prevention practices. Furthermore, identification of differences between men with different ulcer types regarding HIV status and acute HIV infection would be useful for healthcare providers and STI prevention efforts.

Thus, we compared demographics and recent sexual behaviours of men who had either a bacterial or acute HSV-2 ulcer with men who had recurrent HSV-2 ulcers. Finally, we examined the prevalence of HIV sero-positivity and acute HIV among the groups to determine if there was an association between bacterial, or acute HSV-2 ulcers, and newly acquired HIV infection.

\section{Methods}

As part of a larger randomised controlled trial (RCT) on acyclovir therapy conducted in 2006-07, 615 men with GUD aged 1860 years old were recruited from three primary health care clinics in Gauteng Province, South Africa from a total of 635 men who were found to be eligible for the RCT. ${ }^{9}$ We used data from baseline surveys and STI/HIV testing to examine the correlates of: 1) bacterial ulcers vs. recurrent HSV-2 ulcers; and, 2) acute versus recurrent HSV-2 ulcers. The RCT was approved by US Centers for Disease Control and Prevention and University of Witwatersrand ethics review boards. RCT participants provided informed consent. Study details have been previously published. ${ }^{9,10}$

Our diagnostic approach has been described previously. ${ }^{9}$ Briefly, rapid HIV tests [Determine ${ }^{\mathrm{TM}}$ (Abbott Laboratories, USA) and Capillus (Trinity Biotech PLC, Ireland] were used to screen patients at the clinic; discordant results were further tested in the laboratory using three enzyme-linked immunosorbent assays (Bio-Rad, USA; Abbott Murex, UK; and bioMérieux, France). Blood serum from antibody negative participants, who consented to storage of their specimens for future testing, were tested to detect acute infection by HIV RNA PCR. The COBAS AmpliScreen HIV-1 Test v.1.5 (Roche, USA) was used for detection of HIV-1 RNA in pooled samples of 6 specimens and individual specimens. Serological screening was undertaken for syphilis with the rapid plasma reagin (Becton Dickinson and Co., USA) and the Treponema pallidum particle-agglutination (Fujirebio Inc., Japan) assays and HSV-2 IgG (Kalon Biological, UK). ${ }^{11}$ A previously validated multiplex PCR assay was used to test for Treponema pallidum, Haemophilus ducreyi, and HSV. ${ }^{11}$ A PCR assay was used to type HSV positive specimens to differentiate HSV-1 from HSV2 infected lesions. ${ }^{12} \mathrm{~A}$ separate real-time PCR assay for Chlamydia trachomatis L1-L3 was also performed. ${ }^{13}$

Bacterial ulcers were those with Treponema pallidum (syphilis), Haemophilus ducreyi (chancroid), or Chlamydia trachomatis L1-L3 (lymphogranuloma venereum, LGV) detected in the ulcer specimens. Recurrent HSV-2 ulcers were those among men with HSV-2 positive serology and HSV-2 detected in the ulcer. Acute HSV-2 ulcers where those among men with a HSV-2-positive ulcer specimen and HSV-2-negative serology. Among all participants, men with bacterial ulcers were compared to men 
with recurrent HSV-2. Men with acute HSV-2, but did not have a bacterial ulcer, were compared to men with recurrent HSV-2 ulcers. Analyses were conducted for each ulcer outcome measures, separately. Correlates examined included demographics, recent sexual behaviours and HIV test results (HIV sero-positivity or acute HIV). In the absence of anti-HIV antibodies at baseline levels, acute HIV was defined by either detection of HIV RNA at the baseline level or by HIV seroconversion at one month follow-up. ${ }^{6}$ Ulcer size (determined by measurements of largest ulcer) and mean number of ulcers were also included in analyses. Chi-squares, Fisher's Exact tests (acute HIV analyses) and t-tests were used for bivariate analyses. Variables with $p<0.10$ in bivariate analyses were included in adjusted logistic regression models.

\section{Results}

Of the 615 participants with GUD, 7.0\% had a bacterial ulcer ( $n=43), 21.3 \%$ had acute HSV-2 $(n=131), 0.8 \%$ had both a bacterial and acute HSV-2 ulcer $(n=5), 50.1 \%$ had recurrent HSV$2(n=308)$, and $20.8 \%$ had an ulcer of undetermined etiology ( $n$ $=128$ ). Among bacterial ulcers $(n=48), 30$ were attributed to syphilis, 10 to chancroid, and 8 to LGV. For this analysis, we categorised the $0.8 \%(5 / 615)$ of men who had a bacterial and acute HSV-2 ulcer has having a bacterial ulcer. No men had a dually-infected bacterial and recurrent HSV-2 ulcer. Of men who had a bacterial or recurrent HSV-2 ulcer $(n=356)$, men who had a bacterial ulcer were significantly younger than men with recurrent HSV-2 ulcers $(p=0.02)$, and the majority were single (Table 1). Also, men with bacterial ulcers reported more casual sex partners in the past 3 months than men with recurrent HSV-2 ulcers $(p=0.01)$. There were no differences between the groups for other sexual behaviours, in the average time it took men to seek care for their ulcer, or number of ulcers. As compared to men with recurrent HSV-2 ulcers, men with bacterial ulcers had larger ulcers $(p<0.0001)$; and, fewer were HIV-positive (24/48; $50.0 \%$ vs. $237 / 308 ; 77.0 \%, p<0.0001)$. There was no difference in the prevalence of acute HIV between the two groups. In adjusted analyses, men with a bacterial ulcers were significantly more likely to have large ulcers ( $A O R=6.82 ; 95 \% \mathrm{Cl} 3.26$ to 14.29 ) as compared to men with recurrent HSV-2 ulcers. Men with bacterial ulcers were also less likely to test positive for HIV antibodies (AOR $=0.26 ; 95 \% \mathrm{Cl} 0.12$ to 0.55 ).

Of men with HSV-2 who did not have a bacterial ulcer $(n=444)$, $30.6 \%$ had acute herpes $(n=136)$ and $69.4 \%$ had recurrent herpes $(n=308)$. Similar to findings for bacterial versus recurrent HSV-2 ulcers, in bivariate analyses, men with acute HSV-2 were younger $(p<0.0001)$ (Table 2). Conversely, as compared to men with recurrent HSV-2, there were fewer reports of men with acute HSV-2 that had multiple regular sex partners $(p<0.01)$, but also a lower incidence of consistent condom usage with these partners $(p=0.09)$, although this difference was not significant. Note, however, the majority (>75\%) of men in both groups reported multiple regular sex partners in the past 3 months and consistent condom use was remained low $(<20 \%)$. Men with acute HSV-2 did not differ from those with recurrent HSV-2 in ulcer size. However, men with acute HSV-2 sought care more quickly than men with recurrent HSV-2 ulcers $(p=0.01)$. Fewer men with acute HSV-2 tested positive for HIV infection (55/136; $40.4 \%$ vs. $237 / 308 ; 77.0 \%, p<0.0001)$. Although the percent with acute HIV was higher among those with acute HSV-2 $(5 / 81 ; 6.2 \%)$ compared to those with recurrent HSV-2 ulcers $(1 / 71 ; 1.4 \%)$, the difference did not reach statistical significance $(p=0.22)$. In adjusted analyses, men with acute HSV-2 were more likely to be 1825 years $(A O R=6.61,95 \% \mathrm{Cl} 2.96-14.77)$ and were less likely to report multiple regular partners ( $A O R=0.53,95 \% \mathrm{Cl} 0.30-0.96$ ). Finally, as compared to men with recurrent HSV-2, men with acute HSV-2 were less likely to test positive for HIV antibodies at baseline levels (AOR $=0.31,95 \% \mathrm{Cl} 0.19-0.50)$.

\section{Discussion}

Our study found that bacterial infections accounted for a lower proportion of ulcers. Specifically, less than $10 \%$ of participants had a bacterial ulcer but nearly one-third of men had acute HSV2 infection at clinical presentation. Also, we found that men with acute ulcers tended to have larger ulcers than men with recurrent HSV-2 ulcers. HIV positivity at the time of GUD clinical presentation was high among men with bacterial (50\%) and acute HSV-2 ulcers (40\%); however, they were less likely to be infected with HIV as compared to men with recurrent HSV-2 ulcers (77\%). Furthermore, $4 \%$ of men with a bacterial ulcer and $6.2 \%$ of men with acute HSV-2 had acute HIV. Acute and early HIV infection may help drive HIV transmission in sub-Saharan Africa. ${ }^{14}$ Our data emphasise the importance of testing all men with GUD with a rapid and sensitive HIV screening assay for HIV coinfection at the first clinical presentation; ${ }^{15}$ and, to re-test HIV sero-negative men for possible HIV seroconversion at the end of the 'window period' (ideally, 4-6 weeks later).

Other places in Africa, such as a study in Namibia, have found that bacterial infections accounted for a low proportion of ulcers. ${ }^{16}$ In comparison, bacterial ulcers accounted for a higher proportion GUD in etiological studies conducted among men and women in both Malawi in 2004-06 3 and Madagascar in 2011 (D A Lewis, personal communication, 5 January 2015).

Recent sexual behaviours and age were associated with ulcer type, although these findings were not significant in adjusted analyses for bacterial ulcers. We did find that younger men (1834 years old) were more likely to have acute HSV-2 as compared to recurrent HSV-2 in bivariate and adjusted analyses. It is important to note that, across all ulcer groups (bacterial ulcers, acute HSV-2, and recurrent HSV-2), the majority of men reported multiple partners ( 2 or more regular and casual partners) and half reported never using condoms. Thus, given the lack of consistent behavioural and demographic findings across our ulcer groups, a broader rather than targeted approach to the delivery of prevention messages may be useful for men presenting to primary healthcare clinics with GUD. Specifically, it may be useful to discuss STI/HIV prevention efforts with all men who present with GUD. These could include correct and consistent condom use; reduction in number of partners; early treatment of symptomatic STIs; and, the benefits for couples to know their HIV serostatus.

Our study has limitations. The small number of bacterial ulcers precluded examining syphilis, chancroid, and LGV ulcers separately. It is possible that some correlates may vary for the different bacterial ulcer types. The small number of acute HIV infections limits our interpretations of those data. Finally, it is possible that the epidemiology and predictors of the ulcer types have changed over time.

\section{Conclusion}

Our findings emphasise the importance of HIV testing and retesting and strengthening STI/HIV prevention programs for men in South Africa, particularly HIV negative youth that may benefit from prevention services. GUD diagnoses among HIVnegative young adult men can be sentinel for public health opportunities to engage this high risk group in STI/HIV 
Table 1: Characteristics of men with bacterial ulcers as compared to recurrent HSV-2 ulcers, South Africa, 2006-07

\begin{tabular}{|c|c|c|c|c|}
\hline \multirow[b]{2}{*}{ Correlate } & \multicolumn{3}{|c|}{ Bivariate Analyses } & \multirow{2}{*}{$\begin{array}{c}\text { Adjusted Model }(n=354) \\
\text { AOR }(95 \% \mathrm{Cl})\end{array}$} \\
\hline & $\begin{array}{c}\text { Bacterial ulcer }(n=48) \\
n(\%)\end{array}$ & $\begin{array}{l}\text { Recurrent HSV-2 }(n=308) \\
n(\%)\end{array}$ & Unadjusted $\boldsymbol{P}$ value & \\
\hline \multicolumn{5}{|l|}{ Demographics } \\
\hline Age (years) & & & 0.02 & \\
\hline $18-24$ & $13(27.1 \%)$ & $38(12.3 \%)$ & & $1.06(0.33-3.41)$ \\
\hline $25-34$ & $24(50.0 \%)$ & $173(56.2 \%)$ & & $0.99(0.42-2.32)$ \\
\hline 35 and older & $11(22.9 \%)$ & 97 (31.5\%) & & Ref \\
\hline Marital status & & & 0.04 & \\
\hline Married & $7(14.6 \%)$ & $90(29.2 \%)$ & & Ref \\
\hline Cohabitating & $5(10.4 \%)$ & $46(14.9 \%)$ & & $1.29(0.36-4.61)$ \\
\hline Currently single $\mathrm{e}^{\mathrm{a}}$ & $36(75.0 \%)$ & $172(55.8 \%)$ & & $2.57(0.98-6.72)$ \\
\hline Nativity & & & 0.52 & \\
\hline South African & $38(79.2 \%)$ & $229(74.8 \%)$ & & - \\
\hline Other & $10(20.8 \%)$ & 77 (25.2\%) & & - \\
\hline
\end{tabular}

Recent Sexual Behaviours

\begin{tabular}{|c|c|c|c|c|}
\hline $\begin{array}{l}\text { Had multiple regular sex part- } \\
\text { ners, last } 3 \text { months }\end{array}$ & & & 0.36 & \\
\hline No & $9(19.2 \%)$ & $43(14.1 \%)$ & & - \\
\hline Yes & $38(80.9 \%)$ & $263(86.0 \%)$ & & - \\
\hline \multicolumn{3}{|c|}{ Condom use with regular partners ${ }^{b}$} & 0.11 & \\
\hline Never & $20(48.8 \%)$ & $152(55.3 \%)$ & & - \\
\hline Inconsistently & $18(43.9 \%)$ & $80(29.9 \%)$ & & - \\
\hline Always & $3(7.3 \%)$ & $43(15.6 \%)$ & & - \\
\hline \multicolumn{3}{|l|}{ Casual sex partners, last 3 months } & 0.01 & \\
\hline 0 & $25(52.1 \%)$ & $219(71.6 \%)$ & & Ref \\
\hline 1 & $12(25.0 \%)$ & $57(18.6 \%)$ & & $1.29(0.55-3.00)$ \\
\hline 2 or more & $11(22.9 \%)$ & $30(9.8 \%)$ & & $2.10(0.81-5.41)$ \\
\hline \multicolumn{3}{|l|}{ Condom use with casual partners ${ }^{c}$} & 0.95 & \\
\hline Never & $12(52.2 \%)$ & $42(50.6 \%)$ & & - \\
\hline Inconsistently & $7(30.4 \%)$ & $24(28.9 \%)$ & & - \\
\hline Always & $4(17.4 \%)$ & $17(20.5 \%)$ & & - \\
\hline \multicolumn{5}{|l|}{$S T I / H I$} \\
\hline $\begin{array}{l}\text { Mean time to seek care, days } \\
\text { (SD) }\end{array}$ & $8.3(5.0)$ & $7.1(5.3)$ & 0.16 & - \\
\hline Ulcer size & & & $<0.0001$ & \\
\hline $50 \mathrm{~mm}$ or smaller & $13(27.1 \%)$ & $209(67.9 \%)$ & & Ref \\
\hline$>50 \mathrm{~mm}$ & $35(72.9 \%)$ & $99(32.1 \%)$ & & $6.82(3.26-14.29)$ \\
\hline Number of ulcers - mean (SD) & $2.9(2.3)$ & $2.5(2.3)$ & 0.27 & - \\
\hline HIV results & & & $<0.0001$ & \\
\hline HIV-negative & $24(50.0 \%)$ & $71(23.1 \%)$ & & Ref \\
\hline HIV-positive & $24(50.0 \%)$ & $237(77.0 \%)$ & & $0.26(0.12-0.55)$ \\
\hline \multicolumn{5}{|c|}{ Detection of acute HIV infection in men testing HIV-negative at baseline ${ }^{d}$} \\
\hline No & $23(95.8 \%)$ & $70(98.6 \%)$ & 0.42 & - \\
\hline Yes & $1(4.2 \%)$ & $1(1.4 \%)$ & & - \\
\hline
\end{tabular}

$\mathrm{SD}=$ standard deviation. Ns are for adjusted analyses. For adjusted analyses, reference group is "recurrent HSV-2".

aincludes divorced men who are currently single.

bof those who had a regular partner in the past 3 months.

cof those who had a casual partner in the past 3 months ( $n=106)$.

dacute HIV infection - HIV antibody negative test and HIV RNA positive test at baseline or HIV seroconversion (baseline antibody negative, follow-up antibody positive).

prevention counselling or education. It is important that men are equipped with the appropriate knowledge and skills to either prevent, or to seek early treatment for, STIs including GUD.
Furthermore, given the high levels of risk behaviour we observed, it is also important for prevention messages to be disseminated to all men including those with recurrent HSV-2 infections. 
Table 2: Characteristics of men with acute and recurrent HSV-2 ulcers, South African, 2006-07

\begin{tabular}{|c|c|c|c|c|}
\hline \multirow{3}{*}{ Correlate } & \multicolumn{4}{|c|}{ HSV-2 Ulcers } \\
\hline & \multicolumn{3}{|c|}{ Bivariate Analyses } & \multirow{2}{*}{$\begin{array}{c}\text { Adjusted Model }(n=389) \\
\text { AOR }(95 \% \mathrm{Cl})\end{array}$} \\
\hline & $\begin{array}{l}\text { Acute HSV-2 (n=136) } \\
n(\%)\end{array}$ & $\begin{array}{l}\text { Recurrent HSV-2 ( } n=308) \\
n(\%)\end{array}$ & $P$ value & \\
\hline \multicolumn{5}{|l|}{ Demographics } \\
\hline Age (years) & & & $<0.0001$ & \\
\hline $18-24$ & $51(37.5 \%)$ & $38(12.3 \%)$ & & $6.34(2.83-14.22)$ \\
\hline $25-34$ & 72 (52.9\%) & $173(56.2 \%)$ & & $2.37(1.17-4.80)$ \\
\hline 35 and older & $13(9.6 \%)$ & 97 (31.5\%) & & Ref \\
\hline Marital status & & & 0.12 & \\
\hline Married & $29(21.3 \%)$ & 90 (29.2\%) & & - \\
\hline Cohabitating & 17 (12.5\%) & $46(14.9 \%)$ & & - \\
\hline Currently single $^{a}$ & $90(66.2 \%)$ & $172(55.8 \%)$ & & - \\
\hline Nativity & & & 0.27 & \\
\hline South African & 95 (69.9\%) & $229(74.8 \%)$ & & - \\
\hline Other & $41(30.2 \%)$ & 77 (25.2\%) & & - \\
\hline
\end{tabular}

Recent Sexual Behaviours

Had multiple regular sex partners, last 3 months

$$
\text { No }
$$

Yes

Condom use with regular partners ${ }^{\mathrm{b}}$

\section{Never}

Inconsistently

Always

Casual sex partners, last 3 months

0

1

2 or more

Condom use with casual partners

Never

Inconsistently

Always

STI/HIV

Mean time to seek care, days

(SD)

Ulcer size

$50 \mathrm{~mm}$ or smaller

$>50 \mathrm{~mm}$

Number of ulcers - mean (SD)

HIV results

HIV-negative

HIV-positive

Detection of acute HIV infection in men testing HIV-negative at baseline ${ }^{d}$

No

Yes

\begin{tabular}{|c|c|}
\hline $33(24.3 \%)$ & 43 (14.1\%) \\
\hline 103 (75.7\%) & $263(86.0 \%)$ \\
\hline \multicolumn{2}{|c|}{ ners $^{b}$} \\
\hline $74(60.2 \%)$ & $152(55.3 \%)$ \\
\hline 40 (32.5\%) & $80(29.1 \%)$ \\
\hline $9(7.3 \%)$ & $43(15.6 \%)$ \\
\hline \multicolumn{2}{|c|}{ ths } \\
\hline 91 (66.9\%) & 219 (71.6\%) \\
\hline $30(22.1 \%)$ & 57 (18.6\%) \\
\hline 15 (11.0\%) & 30 (9.8\%) \\
\hline \multicolumn{2}{|c|}{$e^{e r s}$} \\
\hline $21(47.7 \%)$ & $42(50.6 \%)$ \\
\hline $11(25.0 \%)$ & $24(28.9 \%)$ \\
\hline $12(27.3 \%)$ & $17(20.5 \%)$ \\
\hline
\end{tabular}

\begin{tabular}{|c|c|}
\hline$<0.01$ & \\
\hline & Ref \\
\hline & $0.53(0.30-0.96)$ \\
\hline \multirow[t]{4}{*}{0.07} & \\
\hline & Ref \\
\hline & $0.86(0.50-1.48)$ \\
\hline & $0.56(0.24-1.32)$ \\
\hline \multirow[t]{4}{*}{0.61} & \\
\hline & - \\
\hline & - \\
\hline & - \\
\hline \multirow[t]{4}{*}{0.67} & \\
\hline & - \\
\hline & - \\
\hline & - \\
\hline
\end{tabular}

\begin{tabular}{|c|c|}
\hline $5.8(3.6)$ & $7.1(5.3)$ \\
\hline $92(67.7 \%)$ & $209(67.9 \%)$ \\
\hline $44(32.3 \%)$ & $99(32.1 \%)$ \\
\hline $2.9(1.9)$ & $2.5(2.3)$ \\
\hline $81(59.6 \%)$ & $71(23.1 \%)$ \\
\hline $55(40.4 \%)$ & $237(77.0 \%)$ \\
\hline & \\
\hline & \\
\hline $5(6.2 \%)$ & $70(98.6 \%)$ \\
\hline
\end{tabular}

\begin{tabular}{|c|c|}
\hline 0.01 & $0.94(0.88-1.00)$ \\
\hline 0.97 & - \\
\hline & - \\
\hline 0.09 & $1.05(0.95-1.16)$ \\
\hline$<0.0001$ & Ref \\
\hline & $0.31(0.19-0.50)$ \\
\hline & \\
\hline & \\
\hline & \\
\hline & \\
\hline
\end{tabular}

$\mathrm{SD}=$ standard deviation. Ns are for adjusted analyses. For adjusted analyses, reference group is "recurrent HSV-2".

aincludes divorced men who are currently single.

bof those who had a regular partner in the past 3 months.

cof those who had a casual partner in the past 3 months $(n=129)$.

dacute HIV infection - HIV antibody negative test and HIV RNA positive test at baseline or HIV seroconversion (baseline antibody negative, follow-up antibody positive). 


\section{Conflict of interest - None}

\section{Notes}

i. The findings and conclusions in this report are those of the authors and do not necessarily represent the official position of the Centers for Disease Control and Prevention.

ii. A portion of these findings were presented at the 19th meeting of the International Society for Sexually Transmitted Disease Research (12/7/2011 in Québec City, Canada).

\section{References}

1. Morgan D, Mahe C, Okongo JM, et al. Genital ulceration in rural Uganda. Sex Transm Dis. 2001;28:431-6.

2. Freeman EE, Weiss $H A$, Glynn JR, et al. Herpes simplex virus 2 infection increases HIV acquisition in men and women: systematic review and meta-analysis of longitudinal studies. AIDS. 2006;20:73-83.

3. Phiri S, Zadrozny S, Weiss HA, et al. Etiology of genital ulcer disease and association with HIV infection in Malawi. Sex Transm Dis. 2013:40:923-8.

4. O'Farrell N, Morison L, Moodley P, et al. Genital ulcers and concomitant complaints in men attending a sexually transmitted infections clinic: implications for sexually transmitted infections management. Sex Transm Disease. 2008;35:545-9.

5. Paz-Bailey G, Rahman M, Chen C, et al. Changes in the etiology of sexually transmitted diseases in botswana between 1993 and 2002: implications for the clinical management of genital ulcer disease. Clin Infect Dis. 2005;41:1304-12.

6. Chen CY, Ballard RC, Beck-Sague CM, et al. Human immunodeficiency virus infection and genital ulcer disease in South Africa: the herpetic connection. Sex Transm Disease. 2006;27:21-9.

7. Freeman EE, Weiss $H A$, Glynn JR, et al. Herpes simplex virus 2 infection increases HIV acquisition in men and women: systematic review and meta-analysis of longitudinal studies. AIDS. 2006;20:73-83.
8. Dangor Y, Ballard RC, L. Exposto F da, et al. Accuracy of clinical diagnosis of genital ulcer disease. Sex Transm Disease. 1990;17:1849.

9. Paz-Bailey G, Sternberg M, Puren AJ, et al. Improvement in healing and reduction in HIV shedding with episodic acyclovir therapy as part of syndromic management among men: a randomized, controlled trial. J Infect Dis. 2009;200:1039-49.

10. Paz-Bailey G, Sternberg M, Lewis DA, et al. Acute HIV infections among men with genital ulcer disease in South Africa. J Infect Dis. 2010;201:1811-5.

11. Lewis DA, Müller EM, Steele L, et al. Prevalence and associations of genital ulcer and urethral pathogens in men presenting with genital ulcer syndrome to primary health care clinics in South Africa. Sex Transm Dis. 2012;39:880-5.

12. Lai W, Chen CY, Morse SA, et al. Increasing relative prevalence of HSV2 infection among men with genital ulcers from a mining community in South Africa. Sex Transm Infect. 2003;79:202-207.

13. Morré SA, Spaargaren J, Fennema JS, et al. Real-time polymerase chain reaction to diagnose lymphogranuloma venereum. Emerg Infect Dis. 2005;11:1311-2.

14. Powers KA, Ghani AC, Miller WC, et al. The role of acute and early HIV infection in the spread of HIV and implications for transmission prevention strategies in Lilongwe, Malawi: a modelling study. Lancet. 2011;378:256-68.

15. WHO and UNAIDS. Guidance on Provider-Initiated HIV Testing and counseling in Health Facilities. World Health Organization, 2007. Available at: http://whqlibdoc.who.int/ publications/2007/9789241595568_eng.pdf.

16. Centre for HIV and STIs, National Institute for Communicable Diseases. Microbiological surveillance for sexually transmitted infections Namibia, 2007 survey. Johannesburg: National Health Laboratory Service; 2007.

Received: 12-03-2015 Accepted: 24-07-2015 